COMUNICAÇÃO CIENTÍFICA

\title{
CARACTERIZAÇÃO BIOMÉTRICA, FÍSICA E QUÍMICA DE FRUTOS DA PALMEIRA BOCAIUVA Acrocomia aculeata (Jacq) Lodd ${ }^{1}$
}

\author{
ELIANA JANET SANJINEZ-ARGANDOÑA² \& CARLOS ALBERTO MACHADO CHUBA ${ }^{3}$
}

RESUMO - Frutos de palmeira bocaiuva (Acrocomia aculeata (Jacq.) Lodd.), coletados em Dourados (MS) e Presidente Epitácio (SP), foram analisados quanto ao aspecto biométrico, a composição química e as características físicas, visando a avaliar seu potencial alimentício. Os frutos de bocaiuva das regiões de procedência não apresentaram diferenças significativas entre si nas características biométricas. O rendimento médio da polpa foi de $42 \%$ em relação ao fruto inteiro, sendo superior ao encontrado na literatura para outros frutos de palmeiras. As determinações físicas e químicas da polpa dos frutos das regiões de Mato Grosso do Sul e São Paulo caracterizam a polpa como sendo pouco ácida (pH 5,70 a 6,29). As amostras de Dourados - MS, foram consideradas mais doces (14,53\% de açúcares redutores totais) e com maior teor de vitamina C (34,57 mg.100 g $\left.\mathrm{g}^{-1}\right)$ em relação às amostras de Presidente Epitácio-SP (11,46 mg.100 g g $\left.^{-1}\right)$. Porém, a intensidade da cor amarelo-laranja foi maior na polpa dos frutos de Presidente Epitácio (SP), sugerindo maior conteúdo de carotenoides.

Termos para indexação: Acrocomia aculeata, macaúba, composição química e física, frutos de palmeiras.

\section{BIOMETRICAL, PHYSICAL AND CHEMICAL CHARACTERIZATION OF BOCAIUVA (Acrocomia aculeata (jacq.) Lodd. ex Mart) PALM FRUITS}

ABSTRACT - Fruits of the Bocaiuva palm - Acrocomia aculeata (Jacq.) Lodd. ex Mart. - collected at Dourados (MS) and Presidente Epitácio (SP), were analyzed in relation to the biometric characteristics chemical composition and physical characteristics, in order to evaluate the food potential. The Bocaiuva fruits showed no significant differences in the biometric characteristics. The average yield of pulp was $42 \%$ compared to the whole fruit, higher than found in the literature for other fruits of palm trees. The physical and chemical characteristics of the fruit pulps from MS and SP regions showed that the pulp was little acid (pH 5.70 to 6.29). Samples from Dourados were sweeter (14.53\% of total reducing sugars) and had higher content of vitamin C (34.57 mg. $\left.100 \mathrm{~g}^{-1}\right)$ than samples from Presidente Epitácio (11.46 mg. $\left.100 \mathrm{~g}^{-1}\right)$. However, the intensity of the yellow-orange was higher in the pulp from Presidente Epitácio indicating higher content of carotenoids in the later.

Index terms: Acrocomia aculeata, macauba, physical - chemical composition, palm fruit.

No bioma Cerrado, encontra-se uma enorme diversidade de plantas frutíferas nativas ou adaptadas com potencial promissor para o aproveitamento agroindustrial. Entre as espécies encontradas, os frutos da palmeira Acrocomia aculeata (Jacq.) Lodd, popularmente conhecida por bocaiuva, bacaiuveira, bacaúva, macaúba, coco-babão, coco-de-catarro, imbocaia, macaíba (ALMEIDA et al., 1998), apresentam atrativos sensoriais, como cor, sabor e aroma peculiares e intensos, ainda pouco explorados na alimentação humana. A bocaiuva está amplamente distribuída em quase todo o Brasil, sendo abundante no Mato Grosso do Sul e Mato Grosso (HIANE et al., 2006, LORENZI et al., 2006) e oeste do Estado de São Paulo-SP. Tradicionalmente, no Pantanal Matogrossense, a comunidade utiliza suas folhas, frutos e sementes como laxante, devido ao efeito purgativo e contra afecções das vias respiratórias. A polpa do fruto é consumida ao natural ou na forma de produtos elaborados, como refrescos, sorvetes, farinhas, entre

${ }^{1}$ (Trabalho 204-10). Recebido em: 28-09-2011. Aceito para publicação em: 24-05-2011.

${ }^{2}$ Doutora em Engenharia de Alimentos, Professor adjunto - Faculdade de Engenharia - Universidade Federal da Grande Dourados/ UFGD - Cx.P. 322 - 79.825-070 - Dourados-MS - eliana.argandona@ufgd.edu.br

${ }^{3}$ Estudante de Engenharia de Produção - Faculdade de Engenharia - Universidade Federal da Grande Dourados- UFGD - DouradosMS -carloschuba@gmail.com / Suporte financeiro: UFGD - FUNDECT/MS 
outros (HIANE et al., 2006). A amêndoa pode ser usada como fonte de matéria-prima para a extração de óleo (ALMEIDA et al., 1998). Seu potencial comercial inclui o uso do óleo para produção de biocombustível e na fabricação de sabão.

O fruto da bocaiuva é uma drupa comestível globosa constituído por epicarpo cartáceo (casca); mesocarpo fino, mucilaginoso e fibroso (polpa) e endocarpo duro e denso (tegumento), contendo semente (amêndoa) adnata ao endocarpo (ALMEIDA et al., 1998). A polpa possui coloração amareloalaranjada, o que sugere a presença de carotenoides, além de outros componentes com valor nutricional, propiciando seu uso na alimentação. No que se refere às características físicas e químicas, reporta-se que é função das condições climáticas, do estádio de maturação, do local de plantio e da época da colheita (PEDRON et al., 2004). A biometria dos frutos fornece informações para detectar a variabilidade genética de populações de uma mesma espécie e as relações com os fatores ambientais. A análise do rendimento de polpa dos frutos fornece subsídios para o aproveitamento agroindustrial (CARVALHO et al., 2003). Assim, informações sobre características biométricas do fruto e semente, bem como sua correlação podem fornecer subsídios para seleção de sementes, aproveitamento das partes comestíveis e estudos de viabilidade econômica dos seus frutos, visando a subsidiar o uso sustentável dessa espécie (CHUBA et. al., 2008).

Do exposto, objetivou-se com este trabalho caracterizar a biometria do fruto, a composição química e física, e o rendimento da polpa dos frutos da bocaiuva colhidos em Dourados (MS) e Presidente Epitácio (SP)

Frutos de Bocaiuva (Acracomia aculeata) foram coletados manualmente nas margens das rodovias próximas às cidades de Presidente Epitácio-SP, e de Dourados-MS. A escolha dos indivíduos para a coleta de aproximadamente 1.000 frutos foi feita de forma aleatória. Os frutos foram transportados ao laboratório de Química da Faculdade de Ciências Exatas e Tecnologia da Universidade Federal da Grande Dourados, onde foram selecionados pelo tamanho e coloração da casca, descartando-se aqueles visualmente danificados. Os frutos foram sanitizados por imersão em solução contendo desinfetante clorado Sumaveg ( $3 \%$ de cloro ativo). Aproximadamente 800 frutos foram divididos em dois lotes de acordo com a procedência, acondicionados em embalagens de polietileno e armazenados a $10^{\circ} \mathrm{C}$.

A massa total do fruto inteiro, o diâmetro externo longitudinal (DEL) e o diâmetro externo transversal (DET) de 20 frutos foram avaliados in- dividualmente em balança analítica (marca AAKer, modelo FA $2104 \mathrm{~N}$ ) e com auxílio de paquímetro digital (marca Digmess). Posteriormente, os frutos foram despolpados para determinar a massa da casca (epicarpo), a massa da polpa (mesocarpo), a massa do endocarpo (tegumento) com semente (amêndoa) e a massa da amêndoa (endosperma). $\mathrm{O}$ rendimento da polpa no fruto da bocaiuva foi determinado subtraindo-se a massa fresca do fruto inteiro.

Uma alíquota de $500 \mathrm{~g}$ de polpa, aleatoriamente separada de cada procedência, foi utilizada nas análises físicas e químicas. $\mathrm{O}$ teor de umidade foi realizado pelo método gravimétrico em estufa (Biopar modelo S81SA) a $70^{\circ} \mathrm{C}$ por 24 horas. O conteúdo de cinzas foi determinado por incineração do material em mufla (marca Quimis-modelo 411 ) a $525^{\circ} \mathrm{C}$ por 3 horas, e a acidez, por titulação com $\mathrm{NaOH}(0,1 \mathrm{~N})$. $\mathrm{O} \mathrm{pH}$ das amostras foi medido por leitura direta, em potenciômetro (modelo PHS-3B), de acordo com os métodos descritos nas Normas Analíticas do Instituto Adolfo Lutz (2005). Os açúcares redutores e totais foram determinados pelo método de Lane-Eynon (AOAC, 1992). Os sólidos solúveis foram mensurados por leitura direta em refratômetro de bancada tipo Abbe (Refratomer 704030 modelo 2waj).

A determinação da atividade de água foi realizada por medida direta em higrômetro Aqualab (modelo CX-2T Decagon Devices Inc., USA) a $25^{\circ} \mathrm{C}$.

A cor das polpas foi avaliada por leitura direta em espectrofotômetro de bancada Cary 50 (marca VARIAN) com o acessório Barrelino ${ }^{+R}$ (Harrick). As leituras foram realizadas no módulo de refletância especular excluída (RSEX), utilizando uma fonte de iluminação D65 e ângulo de detecção de $10^{\circ}$. O sistema de cores utilizado foi CIELAB, com leitura dos parâmetros $\mathrm{L}^{*}$ (luminosidade ou claridade), $\mathrm{a}^{*}$ (índice de saturação verde-vermelho) e $\mathrm{b}^{*}$ (índice de saturação azul-amarelo). As medidas em cada amostra foram realizadas com seis repetições.

$\mathrm{O}$ teor de vitamina $\mathrm{C}$ foi determinado conforme descrito por Benassi et al. (1998). As análises estatísticas foram realizadas com o auxílio do Software Statística 5.0, aplicando-se a análise de variância e o teste de Tukey como teste de média, com nível de 5\%.

As características biométricas dos frutos da bocaiuva coletados nos municípios de Dourados-MS, e Presidente Epitácio-SP, encontram-se na Tabela 1. Os valores médios obtidos para os diâmetros externo longitudinal (DEL) e externo transversal (DET) e para a massa total dos frutos coletados em DouradosMS (34,68 mm, 33,39 $\mathrm{mm}$ e 21,83 g respectivamente), foram maiores que os encontrados para os frutos oriundos de Presidente Epitácio-SP (33, 14 mm, 31,65 mm e 18,86 g, respectivamente). Entretanto, a análise 
de variância mostrou não haver diferença significativa entre as amostras, ao nível de 5\%.

De maneira geral, considerando a média dos valores obtidos nas características biométricas dos frutos coletados em ambos os municípios, a polpa representou aproximadamente $42 \%$ do fruto inteiro, a casca $20 \%$, a semente (Endocarpo com amêndoa) $38 \%$, o endocarpo $31 \%$ e a amêndoa $7 \%$ em relação ao fruto inteiro. Esses valores foram maiores do que os obtidos por Almeida et al. (1998) em frutos coletados na região de Jaboticatubas (MG), onde os autores obtiveram $27 \%$ de polpa, $30 \%$ de casca, $34 \%$ de endocarpo e $7 \%$ de amêndoa, levando a concluir que os frutos de bocaiuva das regiões estudadas neste trabalho apresentaram melhor rendimento em polpa. Ramos et al. (2008), na caracterização de frutos colhidos em Campo Grande-MS, obtiveram resultados semelhantes aos encontrados neste trabalho.

A quantidade da polpa é uma característica importante, refletindo na valorização do extrativismo dos frutos. Neste estudo, foi verificado que cada cacho produziu $6,32 \mathrm{~kg}$ de polpa e $1,36 \mathrm{~kg}$ de endosperma (amêndoa). Considerando uma média de sete cachos por planta, a produção média anual de polpa e de amêndoa poderá alcançar $44,24 \mathrm{~kg}$ e 9,52 kg, respectivamente. Assim, estima-se que 200 plantas por hectare (ha) produzirão $8.848 \mathrm{~kg}$ de polpa e 1.904 $\mathrm{kg}$ de amêndoa. Carvalho et al. (2003), no estudo biométrico dos cachos e frutos da palmeira Najá (Maximiliana maripa (Aublet) Drude) pertencente à mesma família (Arecaceae), obtiveram rendimento de polpa de $14,12 \%$ por fruto, com estimativa de 129,63 $\mathrm{kg}$ de polpa por hectare. Rodrigues et al. (2005) observaram, em frutos das palmeiras patauá (Oenocarpus batauá Mart. ou Jessenia bataua (Mart.) Burret) e najá (Maximiliana maripa (Aublet) Drude, ambas da família Arecaceae, encontradas na Amazônia, relação semente/polpa $(1,16$ e 3,57, respectivamente) bastante elevada, caracterizando-os como frutos com baixo teor de polpa, reforçando a superioridade da bocaiuva (relação semente/polpa de 0,87 a 0,92), mesmo entre palmeiras da mesma espécie. Portanto, a estimativa da produção anual/planta e o percentual de aproveitamento da polpa e do endosperma sugere que a exploração sustentável da bocaiuva pode ser economicamente viável.

As características físicas da polpa, observadas visualmente, mostraram aspecto consistente e fibroso, de cor amarelo-ouro para as amostras de Presidente Epitácio-SP, e amarelo intenso para as amostras de Dourados -MS.

Os resultados das análises físicas, químicas e de cor das amostras de polpa do fruto da bocaiuva das duas regiões estudadas estão apresentados na Tabela
2. O conteúdo de açucares totais encontrados para as amostras das regiões de Presidente Epitácio-SP, e Dourados-SM, foi 11,58\% e 14,53\%, respectivamente (Tabela 2), e estatisticamente não houve diferença significativa $(\mathrm{P}>0,05)$ entre ambas as amostras.

$\mathrm{O}$ conteúdo de água presente na polpa foi maior nas amostras de Presidente Epitácio (52,08\%) em relação às amostras de Dourados $(51,13 \%)$. Dados do NEPA-UNICAMP (2006) apresentam para a mesma espécie teor de umidade de $42 \%$. Para palmeiras da mesma família, Rodrigues et al. (2005) obtiveram valores de umidade de $51,55 \%$ na polpa dos frutos de najá (Maximiliana maripa (Aublet) Drude) e 57,41\% nos frutos de patauá (Oenocarpus batauá Mart. ou Jessenia bataua (Mart.) Burret). Embora esses trabalhos apresentem conteúdo de umidade semelhante aos obtidos neste estudo, o teor de umidade da bocaiuva é ainda menor em relação aos frutos de outras palmeiras, como o açaí, que apresenta $84,73 \%$ de umidade (Pereira et al., 2002) e o butiá, que varia de 84,21 a $86,78 \%$ (MAGRO et al., 2006).

Não obstante a umidade represente a conteúdo de água no alimento, a água pode apresentar-se ligada aos constituintes sólidos do alimento, não podendo ser removida ou utilizada para qualquer tipo de reação; ou pode encontrar-se disponível, favorecendo o crescimento de microrganismos e as reações físicas e químicas. O conhecimento da quantidade de água disponível no alimento (atividade de água) permite prever a estabilidade e a segurança do alimento (RIBEIRO; SERAVALLI, 2007). Assim sendo, apesar de o teor de umidade da polpa da bocaiuva (51 a $52 \%$ ) ser menor que a de frutos de outras palmeiras, a atividade de água da polpa é ainda elevada $(0,90$ a 0,95$)$, podendo ser facilmente contaminada por microrganismos. A atividade de água das amostras de polpa de bocaiuva, de ambos os municípios (Tabela 2), mostrou diferenças significativas entre si, corroborando o teor de umidade obtido. Segundo Munhoz et al. (2007), a polpa de bocaiuva apresenta níveis de água disponíveis relativamente altos ( $a_{w}$ de 0,957$)$, o que vem de encontro com as análises deste trabalho. Alexandre et al. (2004), na caracterização do açaí, obtiveram valores de $\mathrm{a}_{\mathrm{w}} \mathrm{de}$ $0,994 \pm 0,002$, sendo semelhantes aos encontrados para a bocaiuva. Não foram encontrados outros estudos a respeito.

Não houve diferença significativa entre as amostras em relação ao teor de cinzas (Tabela 2) quando comparadas com estudos realizados por NEPA-UNICAMP (2006) e Hiane et al. (2006), e os valores encontram-se próximos aos obtidos por esses pesquisadores $(1,8 \%$ e $1,99 \%$, respectivamen- 
te). Frutos de palmeiras da mesma família botânica, najá e patuá, apresentam teores próximos aos observados (RODRIGUES et al., 2005). Porém, quando comparados com o teor de cinzas encontrado para o açaí $(0,64 \%)$ por Pereira et al. (2002), a macaúba apresenta quantidade maior de cinzas.

No que se refere ao teor de acidez, os valores médios obtidos no presente trabalho variaram de 0,69 a 0,73\% de ácido cítrico. A acidez titulável é um importante parâmetro na apreciação do estado de conservação de um produto alimentício, podendo ser alterado por hidrólise, oxidação ou fermentação. Por outro lado, produtos com alto teor de acidez, no momento do processamento, apresentam melhor conservação, devido à minimização do desenvolvimento de microrganismos (MAGRO et al., 2006). Pereira et al. (2002) obtiveram 0,31\% de acidez em polpa de açaí (palmeira tropical nativa da Amazônia), e Carvalho et al. (2003) relataram acidez de 1,24\% em polpa de bacuri (Platonia insignis Mart.). A análise comparativa com outros frutos mostra que a bocaiuva apresenta menor conteúdo de acidez, conferindo qualidades apropriadas para o consumo in natura.

$\mathrm{O} \mathrm{Ph}$ das amostras de Presidente Epitácio e Dourados apresentou valores de 5,70 e 6,29, respectivamente (Tabela 2). Munhoz et al. (2007) obtiveram $\mathrm{pH}$ de $5,57( \pm 0,030)$ para a polpa de bocaiuva, caracterizando-a como produto pouco ácido. Esses dados são comparáveis ao $\mathrm{pH}$ determinado por Pereira et al. (2002) para polpa de açaí $(5,23)$ (Euterpe oleracea Mart.). Porém, em relação a outras palmeiras, como o butiá (MAGRO et al., 2006), a bocaiuva apresenta baixa acidez, o que favorece suas características sensoriais, tornando-a mais agradável ao paladar, corroborando os resultados obtidos para a acidez titulável.

Em relação ao teor de ácido ascórbico, a amostra oriunda de Dourados apresentou maior conteúdo de vitamina $\mathrm{C}$, sendo obtidos $11,46 \mathrm{mg} .100 \mathrm{~g}^{-1}$, em amostras de Presidente Epitácio-SP e 34,57 mg. $100 \mathrm{~g}^{-1}$ em frutos de Dourados-MS. A análise estatística demonstrou haver diferença significativa $(\mathrm{P}<0,05)$ entre os tratamentos. Dados do NEPAUNICAMP (2006) apresentam para a mesma espécie $13 \mathrm{mg} .100 \mathrm{~g}^{-1}$ de ácido ascórbico. O suco de laranja, considerado fonte de vitamina $\mathrm{C}$, contém de 23,5 mg. $100 \mathrm{~g}^{-1}$ a $76 \mathrm{mg} .100 \mathrm{~g}^{-1}$ (BRANCO et al., 2007; NEPA-UNICAMP, 2006). De acordo com a legislação brasileira, a ingestão diária recomendada (IDR) de vitamina $\mathrm{C}$ para um adulto é de $45 \mathrm{mg}$ (BRASIL, 2005). Assim sendo, a diferença significativa no conteúdo de vitamina $\mathrm{C}$ das amostras analisadas não permite afirmar que a polpa de bocaiuva in natura seja considerada fonte de vitamina C. Entretanto, pode ser fator importante no estudo de melhoramento genético da espécie.

A cor é um atributo fundamental no julgamento da qualidade de um alimento, uma vez que a apreciação visual é o primeiro dos sentidos a ser usado na escolha e aceitação do produto. $\mathrm{Na}$ avaliação instrumental, a cor é especificada numericamente em um espaço tridimensional esférico, definido por três eixos: L*, que varia do preto (0) ao branco (100); a*, do verde ( $-\mathrm{a})$ ao vermelho $(+\mathrm{a})$, e b*, do azul ( - b) ao amarelo (+b), (McGUIRE, 1992).

As amostras analisadas apresentaram predominância da cor amarela b*(Tabela 2). Contudo, em amostras de Presidente Epitácio, observou-se cor amarelo-laranja, com valores encontrados para os parâmetros a* 35,39 e b* de 51,95, e nas amostras de Dourados foram 30,74 e 63,26, respectivamente, favorecendo a cor amarela. Em relação à luminosidade ou claridade ( $\left.\mathrm{L}^{*}\right)$, a amostra de Dourados foi considerada mais clara $(77,08)$, o que corrobora os valores da cromaticidade ( $\left.\mathrm{a}^{*} \mathrm{e} \mathrm{b}^{*}\right)$. A amostra de Presidente Epitácio apresentou menor valor de L* $(70,66)$ devido à predominância da cor amarelo-laranja.

A colorimetria tem sido utilizada para caracterizar a cor de diferentes pigmentos, a exemplo das antocianinas (MONTES et al., 2005), clorofila (SINNECKER et al., 2002) e carotenoides (MELÉNDEZMARTÍNEZ et al., 2003). Neste estudo, a correlação da cor com o conteúdo de carotenoides sugere que quanto mais intensa a cor, maior o conteúdo dessas substâncias denominadas bioativas. Entretanto, há necessidade de mais estudos para confirmar a hipótese.

Agradecemos à Fundação de Apoio ao Desenvolvimento de Ensino, Ciência e Tecnologia do Estado do Mato Grosso do Sul (FUNDECT) e à UFGD. 
TABELA 1 - Características biométricas dos frutos de bocaiuva coletados nos municípios de Dourados MS, e Presidente Epitácio - SP

\begin{tabular}{lcccc}
\hline \multirow{2}{*}{$\begin{array}{c}\text { Características } \\
\text { biométricas }\end{array}$} & \multicolumn{2}{c}{ Dourados-MS } & \multicolumn{2}{c}{ Presidente Epitácio-SP } \\
\cline { 2 - 5 } Fruto - DEL $(\mathrm{mm})$ & $34,68 \pm 1,55$ & 0,04 & $33,14 \pm 1,38$ & CV $(\%)$ \\
Fruto - DET $(\mathrm{mm})$ & $33,39 \pm 1,26$ & 0,04 & $31,65 \pm 1,30$ & 0,04 \\
Fruto $(\mathrm{g})(\mathrm{MT})$ & $21,83 \pm 1,49$ & 0,07 & $18,86 \pm 2,13$ & 0,04 \\
Casca $(\mathrm{g})$ & $4,54 \pm 0,48$ & 0,11 & $3,48 \pm 0,56$ & 0,11 \\
Polpa $(\mathrm{g})$ & $8,98 \pm 1,07$ & 0,12 & $7,90 \pm 1,21$ & 0,16 \\
Endocarpo $(\mathrm{g})$ & $8,31 \pm 0,61$ & 0,07 & $6,85 \pm 0,88$ & 0,15 \\
Endosperma $(\mathrm{g})$ & $1,35 \pm 0,09$ & 0,07 & $1,17 \pm 0,23$ & 0,13 \\
\hline
\end{tabular}

CV, coeficiente de variação.DEL, diâmetro externo longitudinal. DET, diâmetro externo transversal. MT, massa total.

TABELA 2 - Análises físicas e químicas obtida da polpa de frutos de bocaiuva oriundos de Presidente Epitácio (Amostra.A.) e Dourados (Amostra.B.).

\begin{tabular}{|c|c|c|c|c|c|c|}
\hline \multirow{2}{*}{ Características } & \multicolumn{3}{|c|}{ Presidente Epitácio (A) } & \multicolumn{3}{|c|}{ Dourados (B) } \\
\hline & Média & DP & $\mathrm{CV}$ & Média & $\mathrm{DP}$ & $\mathrm{CV}$ \\
\hline Açúcares redutores (\%) & \multicolumn{2}{|c|}{$5,06 \mathrm{a} \pm 0,09$} & 0,018 & \multicolumn{2}{|c|}{$5,59 \mathrm{a} \pm 0,40$} & 0,072 \\
\hline Açúcares Totais (\%) & \multicolumn{2}{|c|}{$11,58 \mathrm{a} \pm 0,89$} & 0,077 & \multicolumn{2}{|c|}{$14,53 \mathrm{~b} \pm 0,30$} & 0,021 \\
\hline Umidade (\%) & \multicolumn{2}{|c|}{$52,08 \mathrm{a} \pm 0,78$} & 0,015 & \multicolumn{2}{|c|}{51,13 a $\pm 0,89$} & 0,017 \\
\hline Cinzas $(\%)$ & \multicolumn{2}{|c|}{$2,22 \mathrm{a} \pm 0,82$} & 0,369 & \multicolumn{2}{|c|}{$1,57 \mathrm{a} \pm 0,48$} & 0,306 \\
\hline Acidez $(\%)$ & \multicolumn{2}{|c|}{$0,69 \mathrm{a} \pm 0,00$} & 0,000 & \multicolumn{2}{|c|}{$0,73 \mathrm{a} \pm 0,00$} & 0,000 \\
\hline $\mathrm{pH}$ & \multicolumn{2}{|c|}{$5,70 \mathrm{a} \pm 0,02$} & 0,003 & \multicolumn{2}{|c|}{$6,29 b \pm 0,04$} & 0,006 \\
\hline Atividade de água & \multicolumn{2}{|c|}{$0,95 \mathrm{a} \pm 0,00$} & 0,001 & \multicolumn{2}{|c|}{$0,90 \mathrm{~b} \pm 0,00$} & 0,001 \\
\hline Vitamina C (mg.100g-1) & \multicolumn{2}{|c|}{$11,46 \mathrm{a} \pm 2,95$} & 0,257 & \multicolumn{2}{|c|}{$34,57 \mathrm{~b} \pm 0,37$} & 0,011 \\
\hline $\mathrm{L}^{*}$ (preto ao branco) & \multicolumn{2}{|c|}{$70,76 \mathrm{a} \pm 1,92$} & 0,027 & \multicolumn{2}{|c|}{$77,08 \mathrm{~b} \pm 1,92$} & 0,025 \\
\hline $\mathrm{a}^{*}$ (do verde ao vermelho) & \multicolumn{2}{|c|}{$35,39 a \pm 1,36$} & 0,038 & \multicolumn{2}{|c|}{$30,74 b \pm 3,63$} & 0,118 \\
\hline $\mathrm{b}^{*}$ (do azul ao amarelo) & \multicolumn{2}{|c|}{$51,95 \mathrm{a} \pm 3,85$} & 0,074 & \multicolumn{2}{|c|}{$63,26 \mathrm{~b} \pm 5,08$} & 0,080 \\
\hline
\end{tabular}

Médias com letras iguais na mesma linha não diferem significativamente entre si ao nível de $5 \%$. DP, desvio padrão. CV, coeficiente de variação.Parâmetros de cor: $a^{*}, b^{*}$ e L* é a luminosidade ou claridade.

\section{REFERÊNCIAS}

ALEXANDRE, D.; CUNHA, R.L.; HUBINGER, M.D. Conservação do açaí pela tecnologia de obstáculos. Ciência e Tecnologia de Alimentos, Campinas, v. 24, n.1, p.114-119, 2004.

ALMEIDA, S. P.; PROENÇA, C. E. B.; SANO, S. M.; RIBEIRO, J. F. Cerrado: espécies vegetais úteis. Planaltina: Embrapa-CPAC, 1998. p. 14-19.

AOAC. Official methods of analysis of AOAC international. $16^{\text {th }}$ ed. Gaitherburg, 1992. v.2, 1141p.
BENASSI, M. T.; ANTUNES, A. J. A comparison of meta-phosphoric and oxalic acids as extractant solutions for the determination of vitamin $\mathrm{c}$ in selected vegetables. Arquivos de Biologia e Tecnologia, Curitiba, v.31, n.4, p. 507-513, 1998.

BRANCO, I.G.; SANJINEZ-ARGANDOÑA, E.J.; SILVA, M.M.; de PAULA, T.M. Avaliação sensorial e estabilidade físico-química de um blend de laranja e cenoura. Ciência e Tecnologia de Alimentos, Campinas, v.27, n.1, p.7-12, 2007.

BRASIL. Resolução RDC n 269, de 22 de setembro de 2005. Aprovar o "Regulamento técnico sobre a ingestão diária recomendada (IDR) de proteína, vitaminas e minerais". Diário Oficial da União, Brasília, 23 set. 2005. 
CARVALHO, J.E.U.; NAZARÉ, R.F.R.; OLIVEIRA, W.M. Características físicas e físico-químicas de um tipo de bacuri (Platonia Insignis Mart.) com rendimento industrial superior. Revista Brasileira de Fruticultura, Jaboticabal, v. 25, p.326-328, 2003.

CHUBA, C. A. M.; TOMMASELLI, M. A. G.; SANTOS, W. L.; SANJINEZ-ARGANDOÑA, E. J. Parâmetros biométricos dos cachos e frutos da bocaiuva. In: CONGRESSO BRASILEIRO DE FRUTICULTURA, 20., 2008. Vitória, Incaper. Anais... CD-ROM.

HIANE, P. A.; BALDASSO, P. A.; MARANGONI, S.; MACEDO, M. L. R. Chemical and nutritional evaluation of kernels of bocaiuva, Acrocomia aculeata (Jacq.) Lodd.. Ciência e Tecnologia de Alimentos, Campinas, v. 26, n3, p. 683-689, 2006.

INSTITUTO ADOLFO LUTZ. Normas Analíticas, Métodos químicos e físicos para análise de alimentos. 4. ed. São Paulo: Instituto Adolfo Lutz, 2005.

LORENZI, G.M.A.C. Acrocomia aculeata (jacq.) Lodd. ex Mart. - Arecaceae: bases para o Extrativismo Sustentável. 2006. Tese (Doutorado em Agronomia)- Universidade Federal do Paraná, Curitiba, 2006.

MAGRO, N. G. D.; COELHO, S. R. M.; HAIDA, K. S.; BERTÉ, S. D.; MORAES, S. S. Comparação físico-química de frutos congelados de Butiá Eriospatha (Mart.) Becc. do Paraná e Santa Catarina - Brasil. Revista Varia Scientia, Cascavel, v.06, n.11, p.33, 2006.

MCGUIRE, R. G. Reporting of objective color measurements. HortScience, Alexandria, v. 27, n. 12, p. 1254-1555, 1992.

MELÉNDEZ-MARTÍNEZ, A. J.; VICARIO, I. M.; HEREDIA, F. J. Application of tristimulus colorimetry to estimate the carotenoids content in ultrafrozen orange juices. Journal of Agricultural and Food Chemistry, Easton, v. 51, n. 25, p. 7266-7270, 2003.
MUNHOZ, C. L.; CHUBA, C. A. M.; CASTRO, D. M.; VIEIRA, M. C;. SANJINEZ-ARGANDONA, E. J. Análise preliminar dos frutos da guavira e da bocaiuva para sua conservação e posterior processamento. In: ENCONTRO DE INICIAÇÃO CIENTÍFICA, 1., 2007. Dourados. Anais... CD-ROM.

NEPA. Núcleo de Estudos e Pesquisas em Alimentação. Tabela brasileira de composição de alimentos: versão II. Campinas: NEPA-UNICAMP, 2006.105p.

PEDRON, F. A.; MENEZES, J. P.; MENEZES; N. L. Parâmetros biométricos de fruto, endocarpo e semente de butiazeiro. Ciência Rural, Santa Maria, v.34, n.2, p.585-586, 2004.

PEREIRA, E. A., QUEIROZ, A. J. DE M.; FIGUEIRÊDO, R. M. F. Massa específica de polpa de açaí em função do teor de sólidos totais e da temperatura. Revista Brasileira de Engenharia Agrícola e Ambiental, Campina Grande, v.6, n.3, p.526-530, 2002.

RAMOS, M.I.L.; RAMOS FILHO, M.M.; HIANE, P.A.; BRAGA NETO, J.A.; SIQUEIRA, E.M.A. Qualidade nutricional da polpa de bocaiuva Acrocomia aculeata (Jacq.) Lodd. Ciência e Tecnologia de Alimentos, Campinas, v. 28, p. 90-94, 2008.

RIBEIRO, E.P.; SERAVALLI, E.A.G. Química de alimentos. 2.ed. São Paulo: Blucher, 2007. p.10-12.

RODRIGUES, A. M. C.; GAMA, S. S.; LINS, R. T.; RODRIGUES, P. R.; SILVA, L.H.M. Estudo da potencialidade de três oleaginosas amazônicas para a produção de biodiesel: biodiesel o novo combustível do Brasil. Belém: Universidade Federal do Pará, Laboratório de Operações de Separação, 2005. p. $345-350$

SINNECKER, P.; GOMES, M. S. O.; AREAAS, J. A. G.; LANFER-MARQUEZ, U. M. Relationship between color (instrumental and visual) and chlorophyll contents in soybean seeds during ripening. Journal of Agricultural and Food Chemistry, Easton, v. 50, n. 14 , p. 3961-3966, 2002.

MONTES, C.; VICARIO, I. M.; RAYMUNDO, M.; FEET, R.; HEREDIA, F. J. Application of tristimulus colorimetry to optimize the extraction of anthocyanins from jaboticaba (Myricia jaboticaba Berg). Food Research International, Barking, v. 38, n. 8-9, p. 983-988, 2005. 